evaluate this case thoroughly to come up with diagnosis of atlanto axial dislocation confirming Grisel syndrome.

Case Summary We present a case of a 2-year girl old who presented with history of fever, on and off neck stiffness and reduced oral intake for three days. She was given IM benzylpenicillin in the community considering possibility of meningitis.

There were similar two distinct episodes in past where she developed cough, fever and transient difficulty in moving her neck. Both times she recovered fully without any residual symptoms.

Her systemic review was unremarkable. She was normally fit \& healthy with up to date immunizations.

On examination, she had head tilt to the right side with diffuse swelling in left mandibular region. There were no rashes. T-37. $4^{\circ} \mathrm{C}$. Other examination were unremarkable.

Apart from marrginally elevated CRP her septic screen was normal. Her persistent symptoms despite intravennous antibiotics prompted CT scan of her neck showing rotatory sublaxation of Atlantoaxial joint suggesting Griesel Syndrome while her MRI brain was reported as normal ruling out space occupying lesion.

A week later her serology confirmed the diagnosis of mumps despite of having previous immunization.

She was transferred to tertiary care hospital where a ASPEN neck collar was inserted under sedation with good recovery.

Discussion While approaching patients with acquired torticollis one should be mindful of wide range of diffrentials like simple trauma to potential space occupying lesion. Blankstein et el describes $61 \%$ cases of torticollis being non traumatic in this age group. Underlying pathology could be very diverse ranging from ADEM to Kawasaki disease.

In our case, as patient presented with fever and diffuse neck swelling, we considered only infective etiology initially. But with her persistent symptoms we expanded our diffrentials and investigated accordingly confirming Griesel syndrome secondary to Mumps. Park et el descibes how pharyngovertebral vein act as an septic channel leading to atlanto axial hyperemia and inturn present as torticollis.

Conclusion Our case is learning lesson to consider possibility of Mumps as diffrential diagnosis irrespetive of previous immunization status and also to remember comorbidities associated with infective patology like Griesel syndrome.

\section{INTUSSUSCEPTION ENCEPHALOPATHY : A CLINICALLY DECEPTIVE PRESENTATION}

Maymunah Khries*, Paul Bellis, Yincent Tse, Leigh McDonald. Great North Children's Hospital

10.1136/archdischild-2021-europaediatrics.57

A 7-month-old presented with reduced responsiveness and non-bilious vomiting. On presentation, he was encephalopathic, apyrexial with normal vital signs. Pupils were intermittently miotic and initial abdominal examination was normal. Investigations including blood gas, biochemistry, inflammatory markers, metabolic and toxicology panels were normal. A CT brain scan showed no abnormality.

Abdominal examination 24 hours later elicited a possibility of tenderness.
Therefore we proceeded with abdominal ultrasound which revealed an evidence of ileocolic intussusception. Initial standard management of ileocolic intussecption was attempted by radiological pneumatic reduction (air enema) which was unsuccessful. Subsequent definitive surgical management achieved by laparotomy and manual reduction successfully released the obstruction.

He recovered uneventfully and underwent usual post-surgical care.

Discussion A recent study showed about $4 \%$ of children diagnosed with intussusception had one or more neurological symptoms recorded at presentation. Lethargy was the most frequent, followed by hypotonia, generalised weakness, paroxysmal events, and fluctuating consciousness. One study reported a series of 13 cases of children whom impairment of the mental state preceded the appearance of common gastrointestinal symptoms. Another distinctive feature is the presence of miosis. The aetiology is unclear but there has been hypotheses that this could be caused by the production of endogenous opioid in response to stress and pain.

Conclusion This case illustrates a rare occasion of intussusception presenting as an acute encephalopathy in the absence of typical signs of bowel obstruction.

Although uncommon, the recognition of this possibility should be entertained, particularly in an unexplained encephalopathy.

\section{COMPARATIVE CHARACTERISTICS OF THE ETIOLOGICAL SPECTRUM OF ACUTE VIRAL RESPIRATORY INFECTIONS IN PEDIATRIC PATIENTS IN UKRAINE DURING 2018- 2020}

VE Khomenko, OV lemets*, OP Volosovets, SP Kryvopustov, TR Umanets, OV Mozyrska. O. O. Bogomolets National Medical University, Kiev, Ukraine

\subsection{6/archdischild-2021-europaediatrics.58}

Background Acute respiratory viral infections (ARVI) continue to be the main reason for seeking pediatric care in Ukraine. Comparative characteristics of etiological spectrum of ARVI in Ukrainehas not been sufficiently studied.

Aim of Study to investigate the etiology of ARVIand compare it between epidemiological seasons 2018-2019 and 2019. 2020.

Methods The study was conducted inthe Eurolab clinic (Kyiv, Ukraine) during the period 2018-2020. Nasopharyngeal swabs, collected from ARVI children aged 2 months to 16 years old, were analyzed by multiplex real time polymerase chain reactionfor 7 viruses - Respiratory Syncytial virus (RSV), Parainfluenza virus (PIV), Adenovirus (AdV), human Metapneumovirus (hMPV), Rhinovirus (RV), human Bocavirus $(\mathrm{hBoV})$ and Coronavirus (CoV). Rapid influenza diagnostic testing was used.

Results 147 samples were collected during the period from October 2018 until February 2019; 125 (85,0\%) were positive: hMPV - 33 (26,3\%), IVA - 28 (22,4\%), RV - 21 $(16,8 \%)$, RSV $-10(8,0 \%), \mathrm{hBoV}-7$ (5,6\%), AdV - 5 $(4,0 \%)$, PIV - $4(3,4 \%)$. Diagnosis of pneumonia was established in $18 \%$ of children infected by hMPV and $21 \%$ of children with IVA infection. Other clinical manifestations of hMPV infection were: tracheobronchitis, obstructive bronchitis, bronchiolitis, rhinopharyngitis, laryngitis.12 of the children with RV infection had symptoms of rhinopharyngitis, 
wheezing, bronchitis and laryngitis. 203 nasopharyngeal swabs were collected during epidemic season 2019-2020, 180 $(88,7 \%)$ of them were positive: RV - 56 (31,7\%), AdV - 39 (22,2\%), CoVNL-63, 229E - 28 (12,7\%), hBoV - 19 $(10,6 \%)$, IVA -15 (8,3\%), PIV - 12 (6,6\%). Confirmed diagnosis of pneumonia had $6(10,6 \%)$ children with RV. 10 $(17,8 \%)$ children with AdV infection had pneumonia as a clinical manifestation. CoVNL-63, 229E caused rhinopharyngitis, croup, wheezing, tracheobronchitis. Generally, 103 samples were collected during the period from March 2019 until September 2019. 98 of them were positive: RSV -37 (37,8\%), RV -29 (29,6\%), CoV - 12 (12,2\%), AdV - 10 (10,2\%), PIV - $9(9,2 \%)$.

Conclusions There has been a change in the etiological spectrum of ARVI during the last two years. During epidemic season 2018-2019 in Ukraine, the most prevalent viruses were hMPV -33 (26,3\%), IVA -28 (22,4\%), RV - 21 (16,8\%). During epidemic season 2019-2020 the most commonlydetected pathogens were RV- 56 (31,7\%), AdV 39 (22,2\%), CoVNL 63, 229E28 (12,7\%).

Epidemiological studies should be continued in order to forecast the probable clinical course of disease, and for optimization therapy.

\section{THE ADOLESCENT SLEEP CRISIS: THE CAUSES, CONSEQUENCES AND SOLUTIONS}

Ffion Byrne*. University of Bristol

10.1136/archdischild-2021-europaediatrics.59

This paper analyzes how reduced sleep duration and quality, referred to as sleep deprivation, affects adolescent development, with a particular interest in associations with obesity and mental health.

To answer this question, a literature search was conducted that produced 12 suitable papers for analysis. These papers were read and reviewed to identify the main findings which were then collated to find common themes.

The results showed that there were five main developmental areas affected by sleep deprivation: academic achievement, obesity, social jetlag, mental health and parent-adolescent relationship.

This study emphasizes the need for greater research into the effects of adolescent sleep deprivation in UK samples. It also highlights three ways in which adolescent sleep deprivation could be prevented: earlier school start times, sleep education and parental education.

\section{IMPACT OF COVID - 19 ON ONCOLOGY PATIENTS IN IRELAND}

Livija Ebner*, Sana Marium Ali, Muhmmad Azam. Wexford General Hospital, HSE

10.1136/archdischild-2021-europaediatrics.60

Background Cancer is a major cause of mortality in Ireland. The total number of cases will almost double by 2040. 1 in 3 people of all age group will develop cancer in their lifetime. On average 137 cases of cancer were diagnosed per year among children under the age 15 during the 21 year period.
211 cases of cancer were diagnosed per year under the age of 20. Five year survival has average $81 \%$ in most recent 10 years. Majority of survivors had been diagnosed with Leukemia $31 \%$. Brain and CNS Tumour's surviving rate is $23 \%$.

Aims To analyse the impact of COVID -19 on Oncology patients. Reason of Hospital admission during COVID-19 Pandemic in 2020-2021 in Wexford General Hospital. To aim for excellent patient care of Oncology patients and to improve access, quality and value for all children with Oncology issue in Wexford area. Assess the resources given to care for these inpatients and changes of workload in last year in COVID 19 Pandemic.

Standards Assessment of COVID-19 impact on Oncology patients if there is any relation to their hospital admission and sick days, has there been any relation of newly diagnosed Oncology patients due to COVID-19.

Difficulties of high risk Oncology patients in Paediatric Ward during COVID-19 Pandemic. The need of properly trained staff to deal with the workload during COVID-19 Pandemic with proper safety and healthcare to the staff and patient. Implementation of new National Cancer Strategy CHI Crumlin following the standards of treatment of Oncology patient according to $\mathrm{CHI}$ guidelines. National Model of care in Paediatric Oncology Patient Services in Ireland.Concept of integrated Multidisciplinary Care.

Methodology A HIPE search was completed for oncology patients admitted in Wexford General Hospital from January 2020 - January 2021 inclusive. All charts were reviewed and appropriateness of inclusion in the Study was confirmed. Following data was collected. Chart review. Date of birth. Age of diagnosis. Date of admission, cause of admission and diagnosis. Length of stay in hospital. Inclusion of Covid 19 PCR test and its outcome of Oncology patients. Newly diagnosed cases. To rule out if there is any correlation of Covid 19 in newly diagnosed and previously diagnosed patients causing them to be admitted in the hospital in Wexford area. Gender and age breakdown of oncology patients. Transfusion rate, most common cause of infection causing increase in number of bed days and hospital admission.

Result Total number of Oncology patient in Wexford area is 20. 9 oncology patients were admitted from January 2020 to January 2021 out of which 6 new and 3 previously diagnosed oncology patient. Total 12 male oncology patients and 8 female patients in total. 6 newly diagnosed oncology patient from( January 2020 to January 2021), out of which 3 ALL( Acute Lymphoblastic Leukemia),1 Pilocytic Astrocytoma,1 Medulloblastoma,1 Wilms tumor respectively. Peripheral blood culture was tested negative for all inpatients except 1 that was likely contaminant sample. Blood transfusion was given only to ALL patients, $46 \%$ pack RBC was transfused and 54\% platelet transfusion was given to patient admitted in Wexford General Hospital in Paeds ward. 6 patients were transferred to Tertiary care hospital as they were newly diagnosed oncology patients and 3 were not transferred as they had recurrent infection treated in local hospital. Total 9 admissions from January 2020 till January 2021, all patients were tested for COVID 19 PCR and all were negative. Total number of bed days occupied are 93 days out of which ALL patients occupied most days i.e 60 days, Medulloblastoma 31 days, Astrocytoma 2 days respectively. 11 times oncology patients admitted with line infection with febrile neutropenia whereas 